\title{
Free Vascularized Second-Toe Distal Interphalangeal Joint Transfer for Reconstruction of Finger Defects
}

\author{
Isao Koshima, MD*; Hirofumi Imai, MD; Syuhei Yoshida, MD; Mitsunobu Harima, MD²; Kensuke \\ Tashiro, MD²; Shuji Yamashita, MD²; Ruben Kannan MD; Tarek Eldahshoury, FRCS ${ }^{4}$ \\ ' International Center for Lymphedema, Hiroshima University Hospital, Hiroshima, Japan \\ ${ }^{2}$ Department of Plastic and Reconstructive Surgery, Graduate School of Medicine, The University of Tokyo, Tokyo, Japan \\ 'Department of Plastic Surgery, Queen Victoria Hospital, East Grinstead, UK \\ ${ }^{4}$ Department of Plastic and Burns, Salisbury District Hospital, Salisbury, UK
}



\begin{abstract}
Objective: Joint defects in the fingers due to osteoarthritis are often repaired with joint implants, arthrodesis or by vascularized proximal interphalangeal joint graft. The disadvantage of grafts, however, is that they may damage the donor toe. As a result of the discussion presented in this paper, we present evidence to indicate that vascularized distal interphalangeal joint transfers from the second toe may be able to be used in the reconstruction of these defects by using microsurgical techniques.

Methods: In this study, we have operated on four cases of hand osteoarthritis using the technique. The distal interphalangeal joint of the second toe was transferred as a composite flap including tendons and/or digital artery perforator flap. There was a follow up period of three to fourteen months after the surgery.

Results: All patients have gained an improved range of motion and stabilization of their joints. There was no skin necrosis or damage to the donor toes, and the function of the remaining proximal interphalangeal joint was preserved.

Conclusion: There is evidence that a distal interphalangeal joint transfer can be a less invasive and effective technique for the reconstruction of traumatic proximal interphalangeal complex defects and advanced carpometacarpal arthritis of the thumb.
\end{abstract}

\section{INTRODUCTION}

Osteoarthritis-related defects of the finger joint can be repaired with temporary replacements, such as implants [1-5], permanent arthrodesis, and vascularized joint grafts like joint flaps or articular flaps [6-11]. Among these methods, the best one is believed to be the permanent substitution through an autogenous vascularized joint transfer utilizing the proximal interphalangeal joint of the second toe [6-11]. This procedure, however, has the disadvantage that the donor toe becomes deformed and shorter, which has the effect of reducing the range of motion.

A supermicrosurgical technique may be used to overcome this disadvantage. In order to minimize the damage resulting from proximal interphalangeal joint transfer, the authors developed a supermicrosurgical technique for dissecting smaller vessels. This technique allows for a less invasive approach to vascularized distal interphalangeal joint transfer of the second toe. We have successfully employed this technique in several reconstructions. The purpose of this paper is to describe four cases of successful transfers of the second toe distal interphalangeal joint composite flap for joint reconstruction of the hand, and so to contribute to the advancement of microanatomy in the field.

\section{METHODS}

From November 1992 to December 2016, we successfully treated four patients who had joint defects in the hand by using the free vascularized distal interphalangeal joint flap from the second toe. The pedicle of each distal interphalangeal joint flap was an articular branch of the digital artery and dorsal cutaneous vein. There were two males and two females in the study, ranging in age from 20 to 54 years old. Joint flaps were transferred in the four cases to replace one distal interphalangeal joint, two proximal interphalangeal joints, and one metacarpophalangeal joint in index finger, index and middle fingers, and one thumb, respectively. Flaps were transferred as a composite flap consisting of joint capsule, tendons, and/or digital artery perforator flaps $[12,13]$. In one case, the proximal interphalangeal joint of the middle finger was repaired with joint transfer and the index finger with first metatarsal artery perforator flap transfer simultaneously from the same donor foot [14].

\section{RESULTS}

The characteristics of the patients were shown in Table 1. After surgery, the patients were followed up for a period ranging from 3 to 14 months. The digital artery perforator flaps that had been transferred had good circulation after surgery, and the recovery process was smooth. In any of the patients involved in this study, there were no serious postoperative complications such as flap necrosis or joint bone absorption.

A high level of joint stability and range of motion was achieved in all cases. All patients in this study had a preoperative range of motion of 0 degrees for the affected finger joints. In three of the four cases, there was a significant improvement in the range of motion of the proximal interphalangeal joints following reconstruction. The patient in case 3 who did not improve his range of motion following distal interphalangeal repair of the affected joint first had preoperative flexor and extensor loss due to osteomyelitis after being bitten by a cat. No necrosis was visible on the donor toes and the remaining proximal interphalangeal joint of the donor toe was still functional. Here we present two cases of free vascularized distal interphalangeal joint flap procedures carried out, along with the outcomes. 


\begin{tabular}{|c|c|c|c|c|c|c|c|}
\hline Case & Age & Sex & Defect & Flap & Follow-up & Complications & poROM \\
\hline 1 & 54 & $\mathrm{~F}$ & Rt Middle PIP loss \& Rt index tendocutaneous defect & DIP joint \& tendons, FMAp flap & 3 months & None & Middle finger $30^{\circ}$; index $45^{\circ}$ \\
\hline 2 & 53 & $\mathrm{~F}$ & Lt thumb CM osteoarthritis & DIP joint \& DAP & 14 months & None & NA \\
\hline 3 & 40 & M & Lt index DIP loss (osteomyelitis \& cat bite) & DIP joint tendon \& DAP & 12 months & None & DIP $0^{\circ}$ \\
\hline 4 & 20 & M & Rt index PIP loss & DIP joint extensor tendon \& DAP & 12 months & None & $30^{\circ}$ \\
\hline
\end{tabular}

CM, carpometacarpal joint; DAP, digital artery perforator flap; DIP, distal interphalangeal joint; F, female; FMAp, first metatarsal artery perforator flap; Lt, left; M, male; NA, not available; PIP, proximal interphalangeal joint; poROM, postoperative range of motion of transferred joint; Rt, right.

\section{Case 1}

It was a 54-year-old woman who had been accidentally injured by an electric saw on the dorsal side of her right index and middle fingers. As a result of contractures of the dorsal skin of the index finger and the loss of extensor tendon, the index finger had a limited range of motion. Furthermore, the patient had a radial shift and poor joint stability of the proximal interphalangeal joint of the middle finger. The condition was caused by a defect in the radial head of the proximal phalanx of the middle finger. A small amount of passive range of motion was observed in both index and middle fingers although there was no active range of motion (Figure $1 \mathrm{~A}-\mathrm{B}$ ).

After 19 months following the injury, reconstructive surgery was performed. The dorsal tendo-cuteneous defect in the index finger was repaired using a first web space tendo-cuteneous flap with a short pedicle that included double perforators of the first metatarsal artery and cutaneous vein (Figure 1C). To thin this flap, subcutaneous fat was sacrificed through dissection to preserve the perforators. The pedicle artery of the flap was interposed into the transected radial digital artery of the index as a flow-through style, and the cutaneous veins of the flap were anastomosed to a vein in the recipient site (Figure 1D).

A minimal amount of proximal interphalangeal joint was resected from the middle finger, and the ensuing proximal interphalangeal joint defect was repaired with a vascularized distal interphalangeal joint that included the flexor and extensor tendons of the second right toe. Stainless steel pins were used to secure the transferred joint using cross pinning. The pedicle of the composite joint flap was joined to the recipient vessels, the articular branch of the radial digital artery $(0.5 \mathrm{~mm}$ in diameter) and a dorsal cutaneous vein. Skin defect caused by bulky joint flap transfer was covered with a split thickness skin graft from the ipsilateral plantar region. The joint defect of the second toe was repaired with an iliac bone graft from the left groin region (Figure 1D).

The postoperative recovery was smooth. At two weeks, joint scintigram measurements confirmed that the flaps had adequate circulation and satisfactory vascularization (Figure 1E-F). The fixed pin was removed two months after surgery. After surgery, the patient's hand returned to its normal appearance three months later. There was also a satisfactory level of movement in the fingers. As can be seen in Figure 1G-I, both the index finger and middle finger have shown improvements in their range of motion. The index finger presented a 45-degree gain in range of motion while the middle finger demonstrated a 30-degree gain. There was an almost normal appearance to the donor toe, as well as a normal proximal interphalangeal function.

\section{Case 2}

The second case involved a 53-year-old woman who was suffering from osteoarthritis of the carpometacarpal joint on her left thumb. The patient had developed severely limited hand function due to debilitating pain, weakness and instability of the thumb, and was suffering for three years. Additionally, she presented a problem with pinching between the thumb and index finger and had difficulties opening bottle caps. The anteroposterior radiograph showed thumb adduction deformity and thumb hyperextension deformity (Figure 2A-B).

Surgery was performed on October 7, 2016. A minimal incision was made, and a superficial resection of the carpometacarpal joint allowed the radial artery and the cutaneous vein to be exposed as recipients at the snuff box. The distal interphalangeal joint complex of the second toe from the left foot was elevated. We preserved the fibular digital neurovascular bundle of the toe so as to preserve the remaining tip of the toe (Figure 2C-D). A cross pin fixation was used for the transfer of the flap to the carpometacarpal joint defect. Pedicle vessels were connected to the recipient vessels in endo-to-side fashion to the radial artery and end-to-end anastomoses to the cutaneous veins at the snuff box area. A donor toe joint was closed and fixed without the need for bone grafting (Figure 2E).

Postoperatively, the recovery course was smooth, and there was good blood flow in the transferred digital artery perforator flap. The bone fixation in the joint was removed two months after the surgery. Despite the shortening of the toe, no signs of skin necrosis were present, and the patient did not have any difficulty walking. The patient's pain and deformity of the thumb diminished after 14 months following surgery. Because of her recovery, the patient was able to continue working full time as a nurse (Figure 2F-I).

\section{DISCUSSION}

As a replacement for conventional vascularized joint grafts, we believe that such terms as joint flap, articular flap, joint composite, complex flap, articulo-cutaneous flap or articulo-tendo-cutaneous flap are more appropriate. There may be clarification in the future regarding the difference between grafts and flaps. Meanwhile, we propose the use of the following terminology: nerve flap versus nerve graft, in the same way that skin flaps are compared to skin grafts.

Based on our experience of free vascularized distal interphalangeal joint flap procedures, we conclude that distal interphalangeal joint flap is a safe and effective procedure for traumatic proximal interphalangeal joint defects without causing damage to the donor toes. This method is deemed superior to conventional proximal interphalangeal joint transfer since less trauma is caused to the donor site [1,6-11].

Approximately $7 \%$ of men and $15 \%$ of women have been observed to have thumb carpometacarpal arthritis on radiographic examination. While most patients experience only minor symptoms, some develop severe pain, weakness, and instability in their thumb, severely impairing their hand function. Treatment consists primarily of removal of the diseased trapezium and stabilization of the metacarpal base $[4,5]$. Surgery may be an option in the case of advanced disease. However, no technique has demonstrated a superior outcome to trapeziectomy yet. Newer treatment options such as ideal prosthetic implants and carpometacarpal arthroplasty are now being used $[4,5]$.

We have successfully completed distal interphalangeal joint transfer for patients with advanced osteoarthritis of the thumb base joint. We believe that 

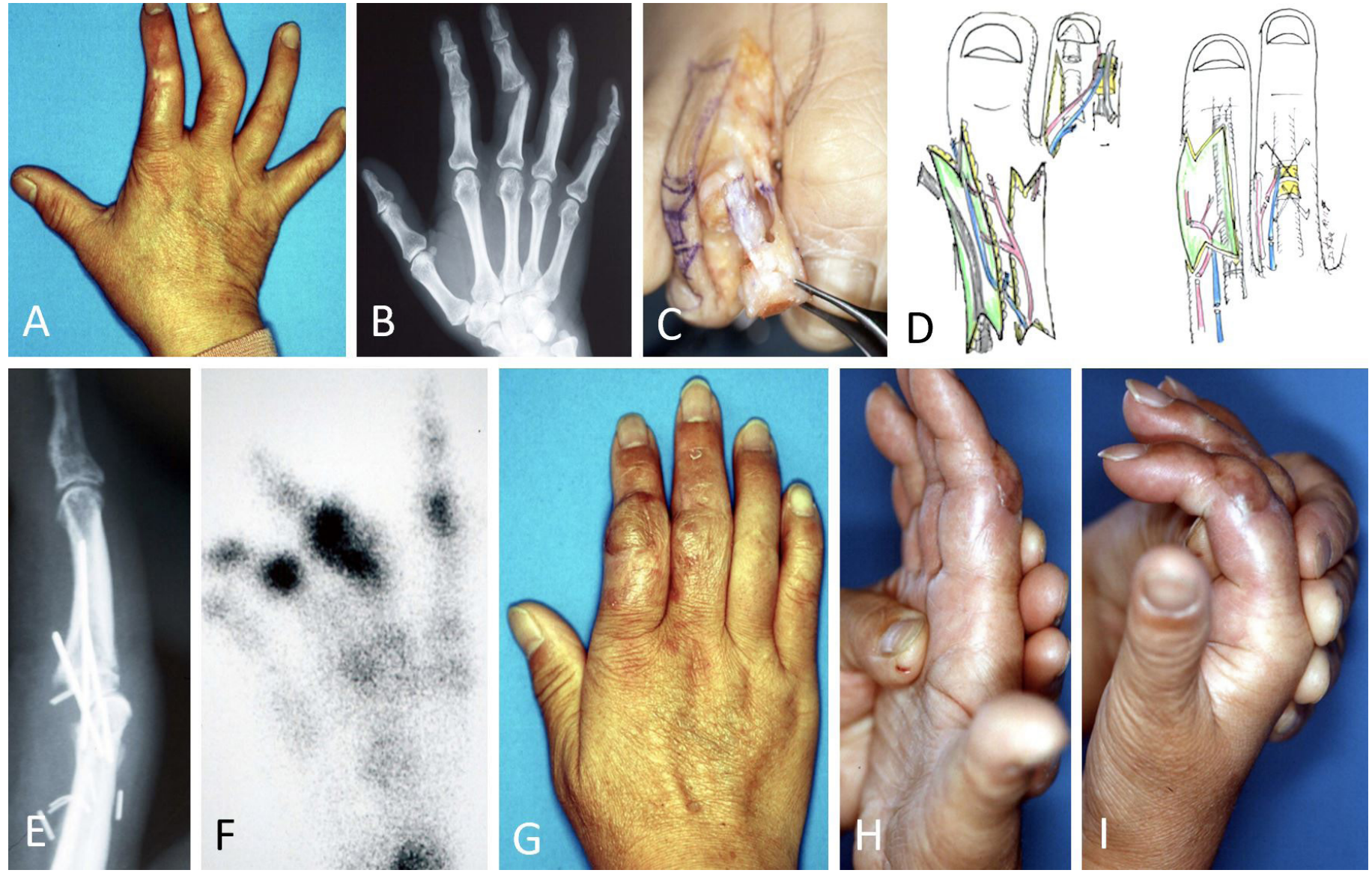

Figure 1. Case 1 presents a 54-year-old woman who accidentally injured both her right index and middle fingers on the dorsal side by an electric saw. (A) A photo showing the preoperative condition of a hand. (B) A radiograph of the hand reveals the loss of the proximal phalanx of the middle finger. (C) The distal interphalangeal tendo-articular flap is elevated while the thin first metatarsal artery perforator tendo-cutaneous flap is elevated in the affected area. (D) Surgical scheme showing flap elevation (on the left) and flap transfers as well as vascular anastomoses with flow-through style applied to digital artery (index finger) and to articular branch of digital artery (middle finger, on the right). (E) The x-ray film of the hand shows the condition of the joint that has been transferred. (F) A bone scintigram reveals the circulation of the joint. (G) An image of the hand three months after surgery taken from the dorsal angle. (H, I) After three months, active range of motion is evident in the proximal interphalangeal joints of the operated fingers.

this method can provide long term relief from pain and/or a truly permanent cure for thumb arthritis. In comparison with previous techniques, this is a novel approach to anatomical reconstructive methods.

Several advantages accompany distal interphalangeal joint transfer, which include permanent joint replacement with autologous well-vascularized bone transfer, avoiding damage to the donor toes compared with proximal interphalangeal joint transfer, and availability of a composite joint complex incorporating flexor and extensor tendon attachments. There are a number of disadvantages to distal interphalangeal joint transfer, including anatomical limitations in range of motion postoperatively as compared to proximal interphalangeal joint transfer, a shorter donor toe after surgery, and technical difficulties when elevating flaps.

The key points of this flap are to avoid damage to the nail matrix to avoid nail deformity and to preserve the circulation of the remaining toe tip and nail by leaving the contralateral neurovascular bundle intact. It is vital to use a fine supermicrosurgical technique to harvest the delicate joint and to dissect the small dorsal vein which needs loupes or an operative microscope.

The first metatarsal artery perforator flap [14] used in case 1 is classified as a capillary perforator flap such as superficial iliac artery perforator flap [15] and thoracodorsal artery capillary perforator flap [16], in which the diameter is less than $0.3 \mathrm{~mm}$. This flap is different from conventional first web space flap, which has the main trunk of the first metatarsal artery as a pedicle. This flap is unique in that it can be made into a thin first metatarsal artery perfo- rator flap through primary thinning, which can be accomplished by removing the fatty tissue. In this way, the perforators of the first metatarsal artery can be preserved. Moreover, as fat is preserved in the web space, the remaining deep peroneal nerve can be protected from postoperative fibrous compression. In addition, tendo-cutaneous flap and flow-through flap are also available as shown in case 1.

Indications for distal interphalangeal joint flap surgery include the reconstruction of minor joint defects such as the proximal interphalangeal joint of the fingers and the interphalangeal joint of the thumb, as well as advanced osteoarthritis of the carpal joints. The repair of complex defects in joints could also be performed with a free joint composite flap including tendons and a mini-digital artery perforator flap with a short flow-through pedicle. Considering future developments, we anticipate that a combination of distal interphalangeal transfer with a first metatarsal artery perforator flap and/or a vascularized deep peroneal nerve flap from the same donor foot can be used for complex hand defects.

\section{CONCLUSION}

Distal interphalangeal joint flap transfer is a reliable technique for reconstructing small finger joints that is less invasive. The technique can be effectively utilized to reconstruct traumatic proximal interphalangeal defects and advanced 



Figure 2. Case 2 presents a 53-year-old woman suffering from osteoarthritis at the carpometacarpal joint on her left thumb. (A) A photo showing the preoperative condition of the hand. (B) An x-ray picture of the hand preoperatively. (C) A distal interphalangeal joint with the digital artery perforator flap elevated. (D) A flap has been transferred to the carpometacarpal joint. (E) On the scheme of reconstruction, it can be seen how the pedicle vessels are anastomosed to the radial artery and the cutaneous veins. (F) An image of the hand nine months after surgery taken from the dorsal angle. (G) It is evident in the nine-month $\mathrm{x}$-ray film that the joints have successfully fused together. (H) Dorsiflexion of the donor toe. (I) Flexion of the donor toe.

carpometacarpal arthritis of the thumb with less invasiveness. A first metatarsal artery perforator flap, classified as a capillary perforator flap, is also an appropriate method for repairing and resolving complex defects in the hand.

\section{ARTICLE INFORMATION}

*Correspondence: Isao Koshima, MD, International Center for Lymphedema, Hiroshima University Hospital, 1-2-3 Kasumi, Minami-ku, Hiroshima City, Hiroshima 734-8551, Japan. Email: koushimaipla@gmail.com

Received: Mar. 11, 2020; Accepted:Jun. 14, 2020; Published: Dec. 30, 2021

DOI: 10.24983/scitemed.imj.2021.00153

Disclosure: It should be noted that a portion of this paper has been presented in part at the 45th Annual Meeting of the Japanese Society of Plastic and Reconstructive Surgery at Sapporo, held in April 1999, and at the Third International Course on Perforator Flap held in Munich in November 1999, as well as the Annual Meeting of the Japanese Society of Reconstructive Microsurgery at the Tokyo, held in April 2005.

Ethics Approval and Consent to Participate: The study is in accordance with the ethical standards of the 1964 Helsinki declaration and its later amendments or comparable ethical standards. The authors obtained permission from the participants in the human research prior to publishing their images or photographs.

Funding: This research received no specific grant from any funding agency in the public, commercial, or not-for-profit sectors.

Conflict of Interest: The authors report no financial or other conflict of interest relevant to this article, which is the intellectual property of the authors.

Copyright (c) 2021 The Author(s). This is an open-access article distributed under the terms of the Creative Commons Attribution 4.0 International License (CC-BY). In accordance with accepted academic practice, anyone may use, distribute, or reproduce this material, so long as the original author(s), the copyright holder(s), and the original publication of this journal are credited, and this publication is cited as the original. To the extent permitted by these terms and conditions of license, this material may not be compiled, distributed, or reproduced in any manner that is inconsistent with those terms and conditions.

Publisher Disclaimer: It is pertinent to note that all opinions and statements made by the author(s) throughout this article are solely those of the author(s). They may not be representative of those of their affiliated organizations, the publishing house, editors, or other reviewers since they are the opinions and statements of the author(s) alone. The publisher does not guarantee or endorse any statements made by the manufacturer of any product mentioned in this article or the author's evaluation.

\section{REFERENCES}

1. Squitieri L, Chung KC. A systematic review of outcomes and complications of vascularized toe joint transfer, silicone arthroplasty, and PyroCarbon arthroplasty for posttraumatic joint reconstruction of the finger. Plast Reconstr Surg 2008;121(5):1697-1707.

2. Badia A. Total joint arthroplasty for the arthritic thumb carpometacarpal joint. Am J Orthop (Belle Mead NJ) 2008;37(8 Suppl 1):4-7.

3. Vogt R, Aerni M, Ampofo C, Schmelzer-Schmied N. [Proximal interphalangeal (PIP) finger prosthesis - what have we learnt? Experiences over 10 years]. Handchir Mikrochir Plast Chir 2012;44(5):293-299.

4. Desai MJ, Brogan DM, Richard MJ, Mithani SK, Leversedge FJ, Ruch DS. Biomechanical comparison of suture-button suspensionplasty and LRTI for basilar thumb arthritis. Hand (NY) 2016;11(4):438-443.

5. Bakri K, Moran SL. Thumb carpometacarpal arthritis. Plast Reconstr Surg 2015;135(2):508-520.

6. Singer DI, O'Brien BM, McLeod AM, Morrison WA, Angel MF. Long-term follow-up of free vascularized joint transfers to the hand in children.J Hand Surg Am 


\section{ORIGINAL}

1988;13(5):776-783.

7. Foucher G, Sammut D, Citron N. Free vascularized toe-joint transfer in hand reconstruction: A series of 25 patients. J Reconstr Microsurg 1990;6(3):201-207.

8. Ishida O, Tsai T-M. Free vascularized whole joint transfer in children. Microsurgery 1991;12(3):196-206

9. Koshima I, Kawada S, Etoh H, Saisho H, Moriguchi T. Free combined thin wraparound flap with a second toe proximal interphalangeal joint transfer for reconstruction of the thumb. Plast Reconstr Surg 1995;96(5):1205-1210.

10. Kimori K, Ikuta Y, Ishida O, Ichikawa M, Suzuki O. Free vascularized toe joint transfer to the hand. A technique for simultaneous reconstruction of the soft tissue. Hand Surg Br 2001;26(4):314-320.

11. Kuzu IM, Kayan RB, Ozturk K, Guneren E. Functional improvement with free vascularized toe-to-hand proximal interphalangeal (PIP) joint transfer. Plast Reconstr
Surg Glob Open 2018;6(7):e1775.

12. Koshima I, Urushibara K, Fukuda N, et al. Digital artery perforator flaps for fingertip reconstructions. Plast Reconstr Surg 2006;118(7):1579-1584.

13. Mitsunaga N, Mihara M, Koshima I, et al. Digital artery perforator (DAP) flaps: Modifications for fingertip and finger stump reconstruction. J Plast Reconstr Aesthet Surg 2010;63(8):1312-1317.

14. Koshima I, Imai H, Yoshida S, Narushima M, Yamashita S, lida T. First metatarsal artery capillary perforator flaps Int Microsurg J 2019;3(1):1.

15. Koshima I, Nanba Y, Tsutsui T, et al. Superficial circumflex iliac artery perforator flap for reconstruction of limb defects. Plast Reconstr Surg 2004;113(1):233-240.

16. Koshima I, Narushima M, Mihara M, et al. New thoracodorsal artery perforator (TAPcp) flap with capillary perforators for reconstruction of upper limb. J Plast Reconstr Aesthet Surg 2010;63(1):140-145. 